\title{
miR-300 promotes proliferation and EMT-mediated colorectal cancer migration and invasion by targeting p53
}

\author{
LIN WANG and PEIWU YU \\ Department of General Surgery and Center of Minimal Invasive Gastrointestinal Surgery, Southwest Hospital, \\ The Third Military Medical University, Shapingba, Chongqing 400038, P.R. China
}

Received April 12, 2016; Accepted May 20, 2016

DOI: $10.3892 /$ or.2016.5193

\begin{abstract}
. p53 mutations in tumors can induce the loss of wild-type tumor-suppressing p53 function, which results in the increase in proliferation, migration and invasion ability in cancer cells. Studies have shown that the expression of p53 is regulated by several microRNAs (miRNAs). In the present study, we found that miR-300 and p53 were significantly increased in colorectal cancer (CRC) tissues when compared with levels noted in adjacent colorectal tissues. Both miR-300 and p53 were significantly correlated with lymphatic metastasis and TNM stage. Both miR-300 and p53 promoted CRC cell (SW480 and HT29) proliferation, migration, and invasion, respectively, in vitro. In addition, we found that miR-300 is a direct positive regulator of p53 through binding to the binding site in the 3'UTR of the p53 gene in human CRC cells. Moreover, both miR-300 and p53 induced CRC cell epithelial-mesenchymal transition (EMT) respectively. Taken together, we demonstrated that miR-300 promoted proliferation and EMT-mediated CRC migration and invasion by targeting p53. These findings provide a new theoretical basis and potential therapeutic targets, and thus lays the foundation for exploring the pathogenesis of CRC.
\end{abstract}

\section{Introduction}

Colorectal cancer (CRC) is one of the most common types of cancers and is a leading cause of morbidity and mortality worldwide (1). It accounts for more than $9 \%$ of the total incidence of malignancies $(2,3)$ and accounted for approximately $8 \%$ of all new cases of malignancies in 2014 (4). Advances in the available methods for CRC treatment have been made. Yet, due to limitations in early cancer detection and accurate prognostic prediction, the survival rates of CRC

Correspondence to: Professor Peiwu Yu, Department of General Surgery and Center of Minimal Invasive Gastrointestinal Surgery, Southwest Hospital, The Third Military Medical University, 30 Gaotanyan Street, Shapingba, Chongqing 400038, P.R. China

E-mail: yupeiwuvip1@163.com

Key words: miR-300, mutant p53, colorectal cancer, proliferation, migration, invasion patients remain low. Thus, identification and knowledge of biomarkers and effective molecular mechanisms regarding $\mathrm{CRC}$ are crucial.

p53 is one of the most significative tumor-suppressor genes. Mutations of p53 result in the loss of wild-type p53 activity (5). These mutant $\mathrm{p} 53$ proteins may also serve as negative inhibitors to play an opposite role of wild-type p53. One study showed that expression of mutant p53 dampened the restoration effect of wild-type p53 (6). These mutant p53 proteins can display new functions in promoting tumorigenesis. Many studies have also shown that p53 mutations act on various human tumors such as colon, breast, liver, and lung cancers (7).

Epithelial-mesenchymal transition (EMT) is the process involved in the regeneration of wound healing, fibrosis and cancer progression (8-11). A large number of studies have shown that the adhesion ability of tumor cells decreases the migration capacity of tumor cells. Thus, EMT is associated with the invasion and metastasis of tumors. In the process of EMT, the expression level of cell adhesion molecules (such as E-cadherin) is reduced while mesenchymal markers such as vimentin protein are increased (12). The EMT process is involved in the invasion, metastasis and drug resistance of tumors.

In the present study, we demonstrated that miR-300 and p53 were significantly increased in CRC tissues. miR-300 and p53 promoted CRC cell proliferation, migration, and invasion in vitro. In addition, miR-300 was found to be a direct positive regulator of $\mathrm{p} 53$ through binding to the binding site in the 3'UTR of the p53 gene in human CRC cells. miR-300 and p53 also induced CRC cell EMT. Taken together, we demonstrated that miR-300 promoted proliferation and EMT-mediated CRC migration and invasion by targeting p53. Our findings aid in the understanding of the underlying mechanisms of CRC metastasis and may elucidate effective therapeutic targets.

\section{Materials and methods}

Cell lines. Human embryonic kidney 293T (HEK293T) cells and human CRC cell lines (NCM460, HT29, SW480, SW620 HCT116 and DLD-1) were purchased from the Type Culture Collection of the Chinese Academy of Sciences (Shanghai, China). NCM460, SW480, HCT116 and DLD-1 cells were cultured in RPMI-1640 medium, and SW620, HT29 and HEK293T cells were cultured in Dulbecco's modified Eagle's 
medium (DMEM) (all from Invitrogen, Carlsbad, CA, USA). Each medium contained 10\% fetal bovine serum (FBS) and $100 \mathrm{U} / \mathrm{ml}$ penicillin and streptomycin (all from Invitrogen). The incubation condition was $5 \% \mathrm{CO}_{2}$ at $37^{\circ} \mathrm{C}$.

Patients and clinical specimens. In the present study, we collected 93 pairs of CRC tissues and matched adjacent noncancerous tissue samples from patients who had undergone surgical treatment for CRC between 2013 and 2016 at Southwest Hospital, Third Military Medical University (Chongqing, China). Written informed consent was provided by each patient. CRC histological diagnosis was assessed according to the World Health Organization (WHO). All tissue samples were washed with sterile phosphate-buffered saline (PBS) and immediately conserved at $-80^{\circ} \mathrm{C}$ until use.

Lentiviral vector construction for p53 expression. For the construction of the lentiviral vector expressing p53, we cloned the human p53 gene from HEK293T cell genomic DNA by PCR with specific designed primers (the forward primercontained aXhol site:5'-CCGCTCGATGGAGGAGCC GCAGTCGA-3', and the reverse primer contained a BamHI site: 5'-CGGGATCCTCAGTCTGAGTCAGGCCCTT-3'). A lentiviral vector expressing EGFP was used as a control. Then, the PCR products were inserted into a lentiviral vector. Packaging vectors (pMD2.G, pMDL-G/P-RRE and pRSVREV) and the transfer vector (p53) were cotransfected into $293 \mathrm{~T}$ cells for $48 \mathrm{~h}$, and the pseudotyped lentiviruses were produced. We performed virus harvest and purification using ultracentrifugation. Thereafter, the SW480 and HT29 cells at a density of 10,000/well were seeded in 24-well plates and transduced with the lentivirus (MOI of 5) and $8 \mu \mathrm{g} / \mathrm{ml}$ polybrene (Sigma-Aldrich, St. Louis, MO, USA).

Transfection of microRNA (miRNA) mimics. A total of $2 \times 10^{5}$ cells were seeded in 6-well plates with antibiotic-free complete medium. The cells were grown overnight, and then transfected with $200 \mu 1$ mature miRNA mimic $(100 \mathrm{nM})$ and Lipofectamine 3000 (Invitrogen) according to the manufacturer's instructions for $72 \mathrm{~h}$. The sequences for miR-300 were 5'-UAUACAAGGGCAGACUCUCUCU-3' (sense) and 5'-AGA GAGAGUCUGCCCUUGUAUA-3' (antisense).

RNA preparation and reverse transcription. According to the manufacturer's instructions, total RNA was extracted from the cells or the CRC tissue samples with TRIzol reagent (Invitrogen). Each $1.0 \mu \mathrm{g}$ of RNA was used as a template to synthesize corresponding cDNA with random primers using a RevertAid First Strand cDNA synthesis kit (Thermo Fisher Scientific, Inc., Waltham, MA, USA).

Quantitative real-time reverse transcription PCR ( $q R T-P C R)$ assay. As previously described (13), we performed all PCR reactions using SYBR-Green PCR Master Mix kit and an ABI 7500 Real-Time PCR system (Applied Biosystems, Warrington, UK). An endogenous housekeeping gene GAPDH was selected as internal loading control. The primer sequences for miR-300 were: miR-300 forward, 5'-TATACAAGGGCA GACTCTCTCT-3' and U6 reverse, 5'-CGCAAGGATGACAC GCAAATTCGT-3'; the primer sequences for p53 were: p53 forward, 5'-GAGGTTGGCTCTGACTGTACC-3' and reverse, 5'-TCCGTCCCAGTAGATTACCAC-3'; the primer sequences for GAPDH were: GAPDH forward, 5'-CATGAGAAGTAT GACAACAGCCT-3' and reverse, 5'-AGTCCTTCCACGATAC CAAAGT- 3 . All results are showed as the mean $\pm \mathrm{SD}$ of three independent experiments.

Cell proliferation. Cell proliferation was detected using the methylthiazol tetrazolium (MTT) assay. Firstly, cells were seeded into 96-well plates at a density of $2 \times 10^{3}$ cells/well with $100 \mu 1$ complete medium and cultured for $24,48,72$, 96 and $120 \mathrm{~h}$. At the specified time of incubation, $20 \mu 1$ of MTT $(5 \mathrm{mg} / \mathrm{ml})$ solution was added to each well and incubation was carried out for $4 \mathrm{~h}$ at $37^{\circ} \mathrm{C}$. The dimethyl sulfoxide (DMSO) was used to dissolve the formazan product for $10 \mathrm{~min}$ at room temperature, and then the absorbance at $490 \mathrm{~nm}$ was measured using a microplate reader. Each condition was determined in quintuplicates, and all experiments were repeated at least 3 times.

Migration and invasion assays. According to the manufacturer's instructions, the migration and invasive abilities of the CRC cells were measured in a 24-well Transwell cell culture chamber (Corning Costar, Inc., Corning, NY, USA) in vitro. Complete medium was added to the lower chamber, and serum-free medium containing $200 \mu \mathrm{l}$ cells $\left(2.5 \times 10^{5} / 100 \mu \mathrm{l}\right)$ was added to the upper chamber. After $24 \mathrm{~h}$ of incubation, the cells with migratory ability migrated through the chamber. The medium in the upper chamber was removed. The cells in the lower chambers were fixed with $4 \%$ paraformaldehyde and stained with $0.1 \%$ crystal violet solution. The number of cells was counted in five areas of constant size per well under the microscope using a 20X objective. Ten microliters 1:3 diluted Matrigel (BD Biosciences, San Diego, CA, USA) was added into the upper chamber for the invasion assays. All experiments were achieved in triplicate.

Western blot analysis. The western blot analysis was carried out as previously described (14). Cells were lysed in a lysis buffer, and the concentrations of total proteins were detected using a BCA Protein Assay kit (Thermo Fisher Scientific, Inc.). Heat-denatured proteins $(30 \mu \mathrm{g})$ were separated and transferred onto polyvinylidene fluoride (PVDF) membranes (Millipore, Billerica, MA, USA). The transferred membranes were incubated with primary antibodies with a proper dilution at $4^{\circ} \mathrm{C}$ overnight. On the following day, the PVDF membranes with proteins were incubated with the secondary antibody. The signal was detected using the enhanced chemiluminescence (ECL) substrate kit and detection system (both from Amersham Biosciences, Piscataway, New Jersey, USA). The primary antibodies mouse anti-E-cadherin $(1: 5,000)$ and mouse anti-vimentin $(1: 4,000)$ were purchased from BD Biosciences. The primary antibody rabbit anti-p53 monoclonal antibody $(1: 1,000)$ was purchased from Abcam (Cambridge, UK). The rabbit anti-GAPDH monoclonal antibody (1:5,000; Cell Signaling Technology, Beverly, MA, USA) was used as an internal control.

Dual-luciferase reporter assay. The database TargetScan (http://www.targetscan.org) was used to predict potential 
A

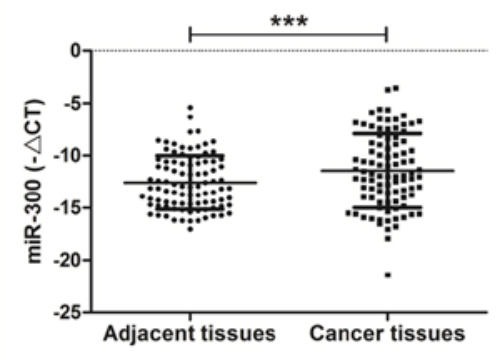

B

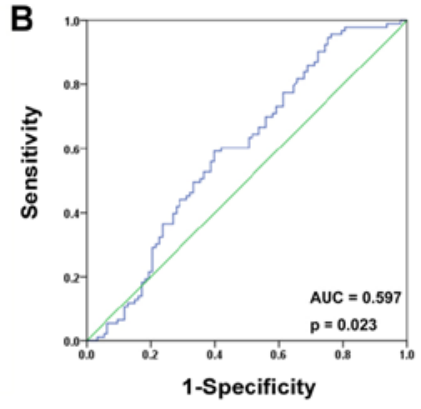

C

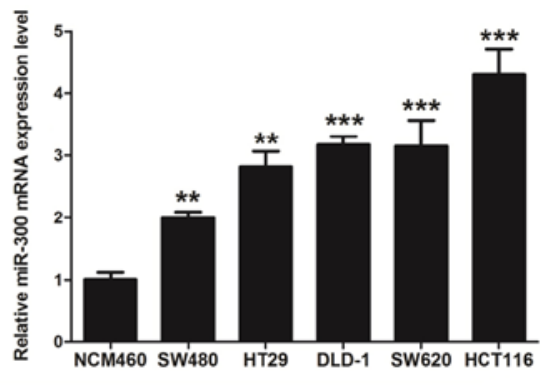

D

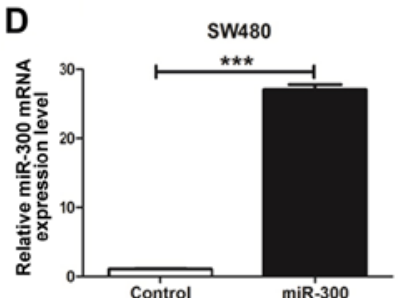

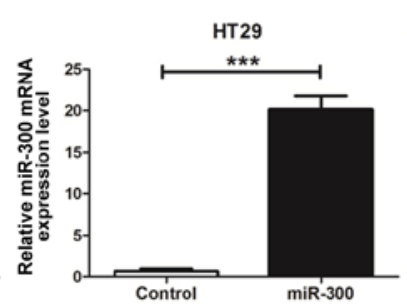

E
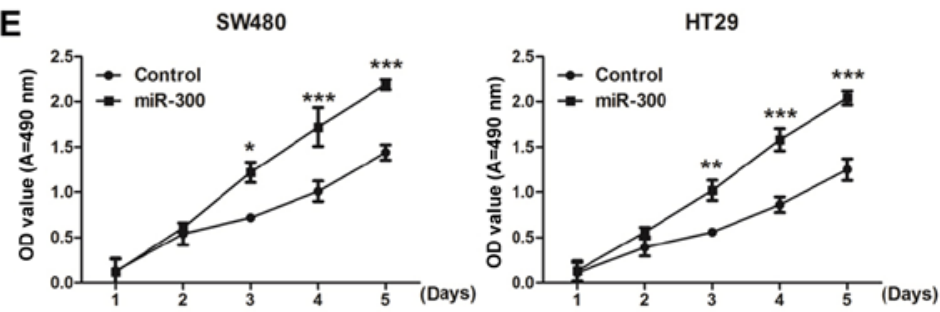

$\mathbf{F}$
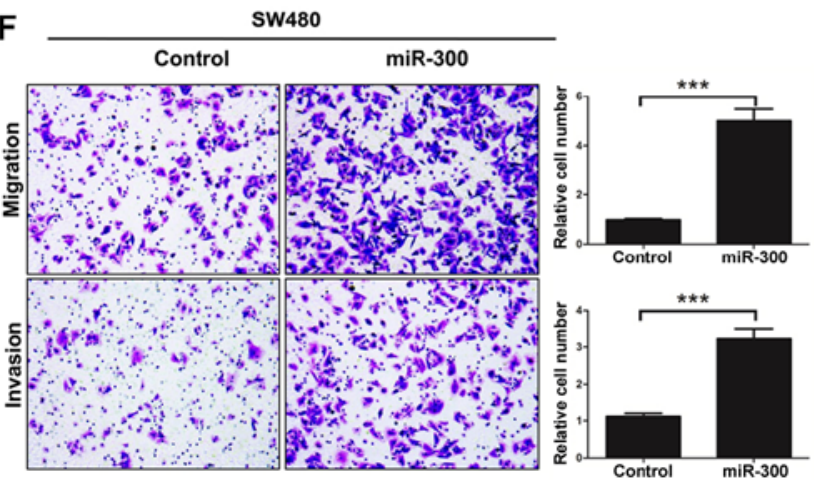

G

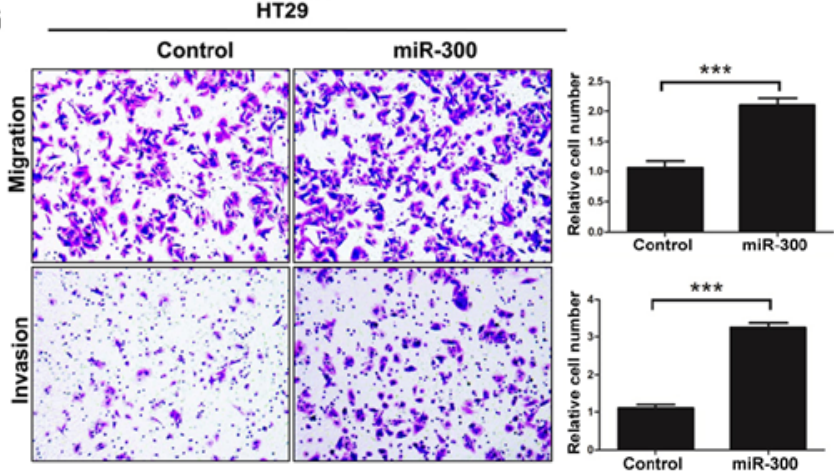

Figure 1. miR-300 is significantly increases in colorectal cancer (CRC) tissues and promotes CRC cell proliferation, migration, and invasion in vitro. (A) Quantitative RT-PCR (qRT-PCR) was used to detected the the mRNA expression level of miR-300 in CRC tissues and adjacent colorectal mucosal tissues. (B) Receiver operating characteristic (ROC) curve indicates the prediction of prognosis for performance of miR-300. (C) The mRNA expression level of miR-300 was detected by qRT-PCR in human-derived colonic epithelial NCM460 cells and CRC cell lines (SW480, HT29, DLD-1, SW620, and HCT116). (D) The expression of miR-300 was detected after SW480 and HT29 cells were transfected with miR-300 or control. (E) The MTT assay was used to determine the ability of proliferation in the SW480 and HT29 cells transfected with miR-300 or control. (F) Transwell assays of SW480 cells transfected with miR-300 or negative control were performed. Magnification, x200; scale bar, $10 \mu \mathrm{m}$. (G) Transwell assays of HT29 cells transfected with miR-300 or negative control were performed. Magnification, $\mathrm{x} 200$; scale bar, $10 \mu \mathrm{m}$. All data are expressed as mean $\pm \mathrm{SD}$ of three independent experiments $\left({ }^{*} \mathrm{P}<0.05,{ }^{* *} \mathrm{P}<0.01,{ }^{* * *} \mathrm{P}<0.001\right)$.

targets for miR-300. DNA fragments of the TP53 3'UTR containing the putative miR-300 binding site (or mutated miR-300 binding site) were amplified by PCR from HEK293T cell genomic DNA and inserted into the PmeI/SpeI sites of the firefly luciferase coding region of the pMIR-report vector (Invitrogen Life Technologies). The plasmids were named wild-type (pMIR-report-p53-wt) and mutated (pMIR-report-p53-mut) sequences, respectively. Mutation from AGCCAG to CACUAU was introduced in the potential miR-300 binding sites. HEK293T cells $\left(3 \times 10^{5}\right)$ were seeded in a 24-well plate and cotransfected with pMIR-report-p53 3'UTR-wt or pMIR-report-p53 3'UTR-mut and miR-300 expression plasmids (a Renilla plasmid as internal reference) using Lipofectamine 3000 for $48 \mathrm{~h}$. The luciferase activities were assessed using the Dual-Luciferase Reporter assay kit (Promega Corp., Madison, WI, USA) according to the manufacturer's instructions. Each transfection was repeated twice in triplicate.
Statistical analysis. SPSS 15.0 software was used to analyze the data. The differences between individual groups were analyzed by t-tests. The Pearson's correlation algorithm was used to analyze the correlation coefficient and the significance between the expression of miR-300 and p53 mRNA. All data are shown as the means $\pm \mathrm{SD}$. $\mathrm{P}<0.05$ was considered to indicate a statistically significant result.

\section{Results}

miR-300 is significantly increased in CRC tissues compared with that in adjacent colorectal tissues. The expression level of miR-300 was detected in 93 pairs of CRC tissues and corresponding adjacent colorectal mucosal tissues by qRT-PCR. Our results demonstrated that the expression of miR-300 in CRC tissues was significantly higher than that observed in the corresponding adjacent colorectal mucosal tissues $(\mathrm{P}<0.001$; Fig. 1A). Our results also showed that miR-300 
Table I. Correlation analysis between the expression level of miR-300 and clinicopathological characteristics of the colorectal cancer patients.

\begin{tabular}{lcrl}
\hline Characteristics & $\begin{array}{c}\text { No. of patients } \\
\text { n }(\%)\end{array}$ & $\begin{array}{c}\text { miR-300 } \\
(\text { mean } \pm \text { SD) }\end{array}$ & P-value \\
\hline Total no. of patients & $93(100.0)$ & & \\
Age (years) & & & \\
$>60$ & $51(54.8)$ & $10.11 \pm 1.88$ & 0.267 \\
$\leq 60$ & $42(45.2)$ & $9.64 \pm 1.62$ & \\
Gender & & & \\
Male & $46(49.5)$ & $9.96 \pm 1.97$ & 0.750 \\
Female & $47(50.5)$ & $9.82 \pm 1.38$ & \\
Invasion & & & \\
T0-T2 & $41(44.1)$ & $9.68 \pm 1.70$ & 0.345 \\
T3-T4 & $52(55.9)$ & $10.09 \pm 1.84$ & \\
Lymphatic metastasis & & & \\
N0 & $62(66.7)$ & $10.84 \pm 2.60$ & $\mathbf{0 . 0 1 3}$ \\
N1-N3 & $31(33.3)$ & $9.13 \pm 1.06$ & \\
Distal metastasis & & & \\
M0 & $71(76.3)$ & $9.87 \pm 1.80$ & 0.472 \\
M1 & $22(23.7)$ & $10.47 \pm 1.38$ & \\
TNM stage & & & \\
0, I and II & $62(66.7)$ & $10.84 \pm 2.60$ & $\mathbf{0 . 0 1 3}$ \\
III and IV & $31(33.3)$ & $9.13 \pm 1.06$ & \\
\hline
\end{tabular}

${ }^{a}$ Significant difference.

was significantly correlated with lymphatic metastasis and TNM stage $(\mathrm{P}=0.013)$ by clinicopathological analysis, but it was not significantly correlated with age, gender, invasion, and distal metastasis ( $\mathrm{P}>0.05$; Table I). Furthermore, in our study, the area under the ROC curve of miR-300 was 0.597 , $\mathrm{P}=0.023$ (Fig. 1B).

miR-300 promotes $C R C$ cell proliferation, migration, and invasion in vitro. The effects of miR-300 on the capacity of CRC cell growth, migration, and invasion were investigated. The mRNA expression level of miR-300 was detected in colonic epithelial NCM460 cells and CRC cell lines (SW480, HT29, DLD-1, SW620, and HCT116) by qRT-PCR. The expression level of miR-300 in the CRC cell lines was significantly higher than that noted in the NCM460 cells (Fig. 1C). SW480 and HT29 cell lines were transfected with miR-300 or control vectors. Overexpression of miR-300 observably increased the expression of miR-300 (Fig. 1D). The cell proliferation capacity of the SW480 and HT29 cells transfected with miR-300 was significantly upregulated compared with the control group as detected by MTT assay (Fig. 1E). The cell migration and invasion capacities of the SW480 cells transfected with miR-300 were also significantly upregulated as assessed by Transwell assays in vitro (Fig. 1F). Similar results were found in the HT29 cells (Fig. 1G). Taken together, we indicated that miR-300 promotes CRC cell proliferation, migration and invasion in vitro.
Table II. Correlation analysis between the expression level of p53 and clinicopathological characteristics of the colorectal cancer patients.

\begin{tabular}{|c|c|c|c|}
\hline Characteristics & $\begin{array}{c}\text { No. of patients } \\
n(\%)\end{array}$ & $\begin{array}{c}\text { p53 } \\
(\text { mean } \pm \mathrm{SD})\end{array}$ & P-value \\
\hline Total no. of patients & $93(100.0)$ & & \\
\hline \multicolumn{4}{|l|}{ Age (years) } \\
\hline$>60$ & $56(60.2)$ & $12.97 \pm 2.62$ & 0.441 \\
\hline$\leq 60$ & $37(39.8)$ & $13.55 \pm 2.04$ & \\
\hline \multicolumn{4}{|l|}{ Gender } \\
\hline Male & $49(52.7)$ & $13.34 \pm 2.34$ & 0.098 \\
\hline Female & $44(47.3)$ & $12.67 \pm 2.77$ & \\
\hline \multicolumn{4}{|l|}{ Invasion } \\
\hline T0-T2 & $42(45.2)$ & $13.53 \pm 2.41$ & 0.573 \\
\hline T3-T4 & $51(54.8)$ & $12.79 \pm 2.54$ & \\
\hline \multicolumn{4}{|l|}{ Lymphatic metastasis } \\
\hline No & $52(55.9)$ & $13.43 \pm 2.40$ & $0.0190^{\mathrm{a}}$ \\
\hline $\mathrm{N} 1-\mathrm{N} 2$ & $41(44.1)$ & $12.69 \pm 2.58$ & \\
\hline \multicolumn{4}{|l|}{ Distal metastasis } \\
\hline M0 & $76(81.7)$ & $13.18 \pm 2.52$ & 0.078 \\
\hline M1 & $17(18.3)$ & $12.21 \pm 2.00$ & \\
\hline \multicolumn{4}{|l|}{ TNM stage } \\
\hline 0, I and II & $52(55.9)$ & $13.43 \pm 2.40$ & $0.0190^{a}$ \\
\hline III and IV & $41(44.1)$ & $12.69 \pm 2.58$ & \\
\hline
\end{tabular}

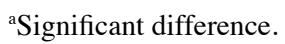

p53 is significantly increased in CRC tissues compared with that in adjacent colorectal mucosal tissues. The expression level of p53 was detected in 93 pairs of CRC tissues and corresponding adjacent colorectal mucosal tissues by qRT-PCR. Our results demonstrated that the expression of p53 in CRC tissues was significantly higher than that observed in the corresponding adjacent colorectal mucosal tissues $(\mathrm{P}<0.001$; Fig. 2A). Our results also showed that p53 was significantly correlated with lymphatic metastasis and TNM stage $(\mathrm{P}=0.019)$ by clinicopathological analysis, but it was not significantly correlated with age, gender, invasion, and distal metastasis ( $\mathrm{P}>0.05$; Table II). Furthermore, in our study, the area under the ROC curve of miR-300 was 0.635 , $\mathrm{P}=0.002$ (Fig. 2B).

p53 promotes CRC cell proliferation, migration, and invasion in vitro. The effects of p53 on the capacity of CRC cell growth, migration, and invasion were investigated. SW480 and HT29 cell lines were transfected with p53 or control vectors. Overexpression of p53 observably increased the mRNA and protein expression levels of p53 (Fig. 2C and D). The cell proliferation capacity of SW480 and HT29 cells transfected with p53 was significantly increased compared with the control group as detected by MTT assay (Fig. 2E). The cell migration and invasion capacities of SW480 and HT29 cells transfected with p53 were also significantly enhanced as detected by Transwell assays in vitro (Fig. $2 \mathrm{~F}$ and $\mathrm{G}$ ). Taken together, 
A

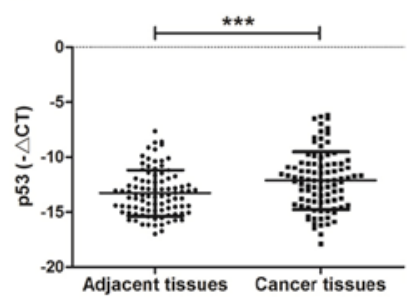

B

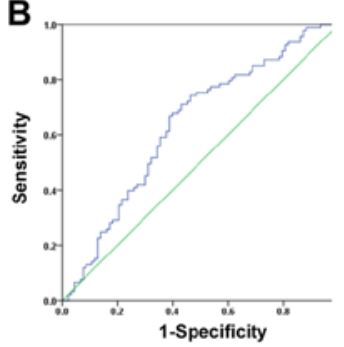

C

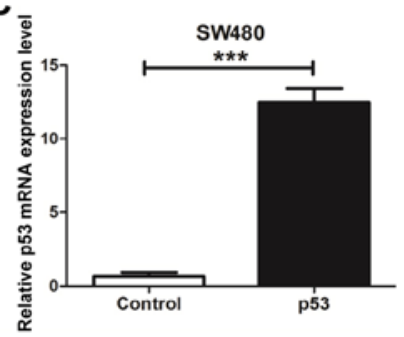

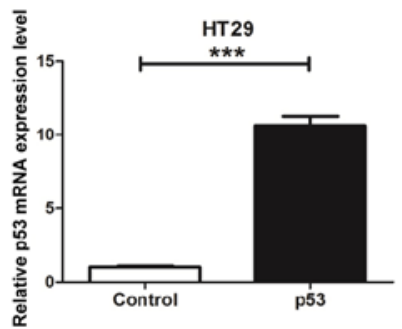

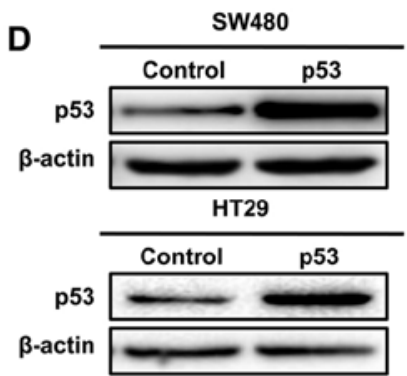

E

SW480

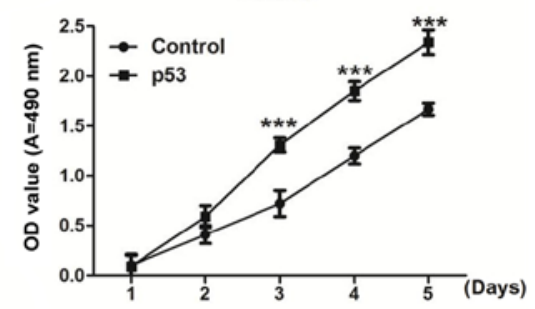

HT29

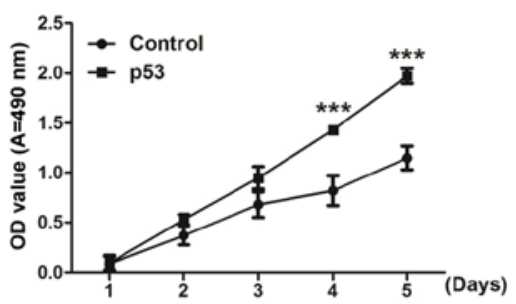

$\mathbf{F}$

SW480

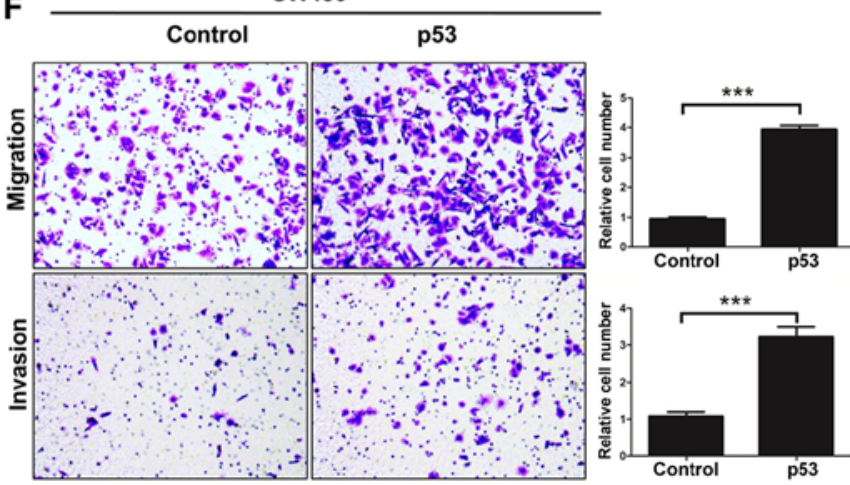

G
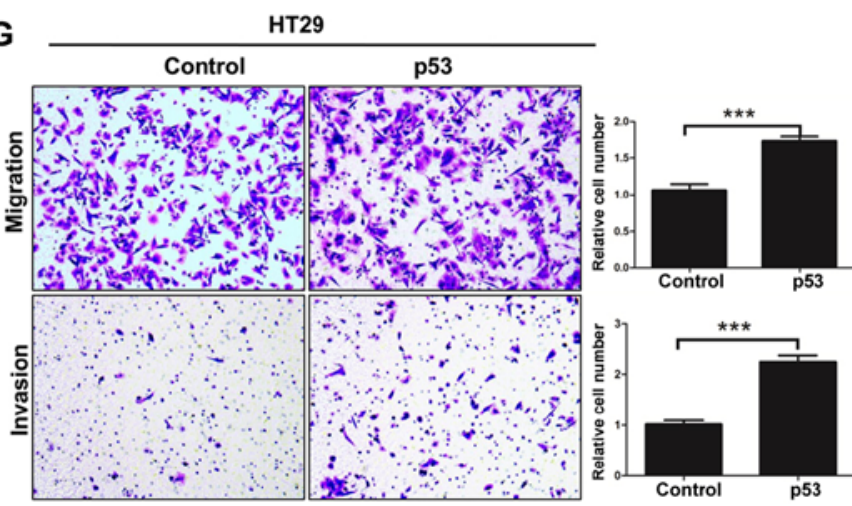

Figure 2. p53 is significantly increased in colorectal cancer (CRC) tissues and promotes CRC cell proliferation, migration and invasion in vitro. (A) qRT-PCR was used to detected the mRNA expression level of p53 in CRC tissues and adjacent colorectal mucosal tissues. (B) Receiver operating characteristic (ROC) curve indicates the prediction of prognosis for performance of p53. (C) The mRNA expression level of p53 was detected by qRT-PCR in the SW480 and HT29 cells transfected with p53 or control. (D) The expression level of p53 protein was assayed by western blot analysis in the SW480 and HT29 cells transfected with equal concentrations of $\mathrm{p} 53$ vectors (EGFP as a negative control). GAPDH antibody acted as a loading control. (E) The MTT assay was used to determine the ability of proliferation in the SW480 and HT29 cells transfected with p53 or control. (F) Transwell assays of the SW480 cells transfected with p53 or negative control were performed. Magnification, x200; scale bar, $10 \mu \mathrm{m}$. (G) Transwell assays of HT29 cells transfected with p53. Magnification, x200; scale bar, $10 \mu \mathrm{m}$. All data are expressed as mean $\pm \mathrm{SD}$ of three independent experiments $\left({ }^{* * *} \mathrm{P}<0.001\right)$.

we demonstrated that p53 promotes CRC cell proliferation, migration and invasion in vitro.

miR-300 regulates p53 gene transcription in human CRC cell lines. We investigated whether miR-300 physically interacts with p53. The correlation between miR-300 and p53 gene expression in CRC tissues was assayed by qRT-PCR. The results indicated that there was a positive correlation between miR-300 and p53 gene expression in the CRC tissues $\left(\mathrm{P}<0.0001, \mathrm{R}^{2}=0.2268\right.$; Fig. $\left.3 \mathrm{~A}\right)$. We indicated that overexpression of p53 increased the mRNA expression level of miR-300 by qRT-PCR assay in the SW480 and HT29 cells transfected with p53 or control (Fig. 3B). qRT-PCR and western blot analysis were used to detect the relative expression level of p53 in the SW480 and HT29 cells transfected with miR-300 or the control. The results indicated that overexpression of miR-300 increased the mRNA and protein expression of p53 (Fig. 3C). Next, we hypothesized that miR-300 may directly regulate the transcriptional level of p53. We cloned the promoter region of wild-type p53 and mutant p53 into the pMIR-report. The results showed that miR-300 increased the promoter activity of p53 in HEK293T cells by luciferase reporter gene assays (Fig. 3D). Taken together, we indicated that miR-300 regulates p53 gene transcription in human CRC cell lines.

Expression levels of p53 influences CRC cell EMT. We further investigated whether miR-300 and p53 influence CRC cell EMT. Western blot analysis showed that the level of E-cadherin was decreased and the levels of the p53 and vimentin were increased after overexpression of miR-300. The level of E-cadherin was increased and the levels of p53 and vimentin were decreased after silencing of p53 by siRNAs compared with the SW480 cells transfected with miR-300 separately (Fig. 4A). Similar results were found in the HT29 cells (Fig. 4B). 

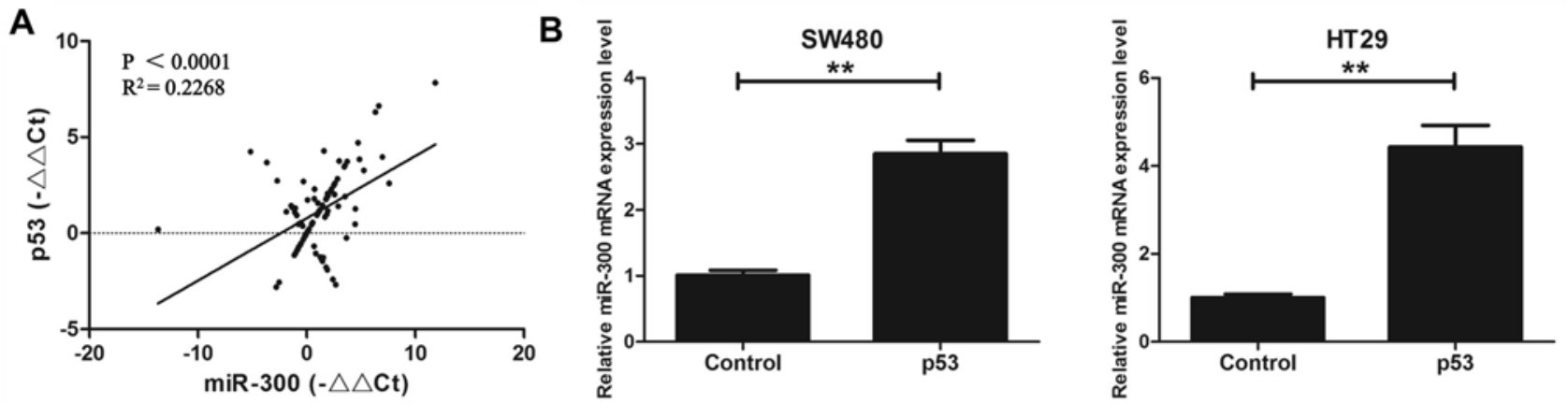

D PMIR-report -p53 3'-UTR
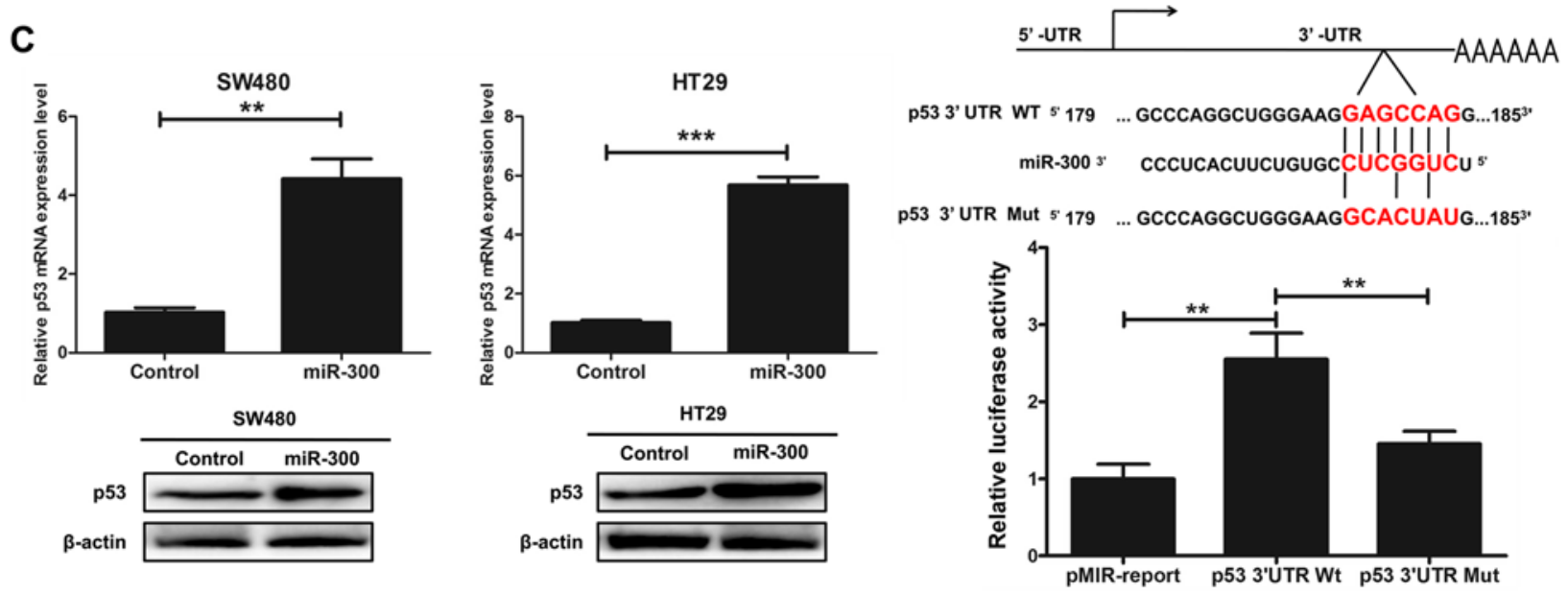

Figure 3. miR-300 regulates p53 gene transcription in mutant p53-expressing human colorectal cancer (CRC) cell lines. (A) Positive correlation between miR-300 and p53 gene expression in CRC tissues was assayed by qRT-PCR. (B) Overexpression of p53 increased the expression of miR-300. qRT-PCR detected the relative expression levels of miR-300 in SW480 and HT29 cells transfected with p53. (C) Overexpression of miR-300 increased the expression of p53. qRT-PCR and western blot analysis were used to detect the relative expression levels of p53 in the SW480 and HT29 cells transfected with miR-300. (D) Overexpression of miR-300 promoted the activity of a luciferase reporter containing p53 3'UTR wild-type. The binding site of miR-300 targeting p53 $3^{\prime}$ UTR (Wt) and its mutant sequences (Mut) are shown (the sites without connections indicate mutation position). Luciferase activity of p53 3'UTR in human embryonic kidney 293T (HEK293T) cells co-transfected with miR-300 plasmids was assayed for 48 h. ${ }^{* * *} \mathrm{P}<0.01,{ }^{* * * *} \mathrm{P}<0.001$.

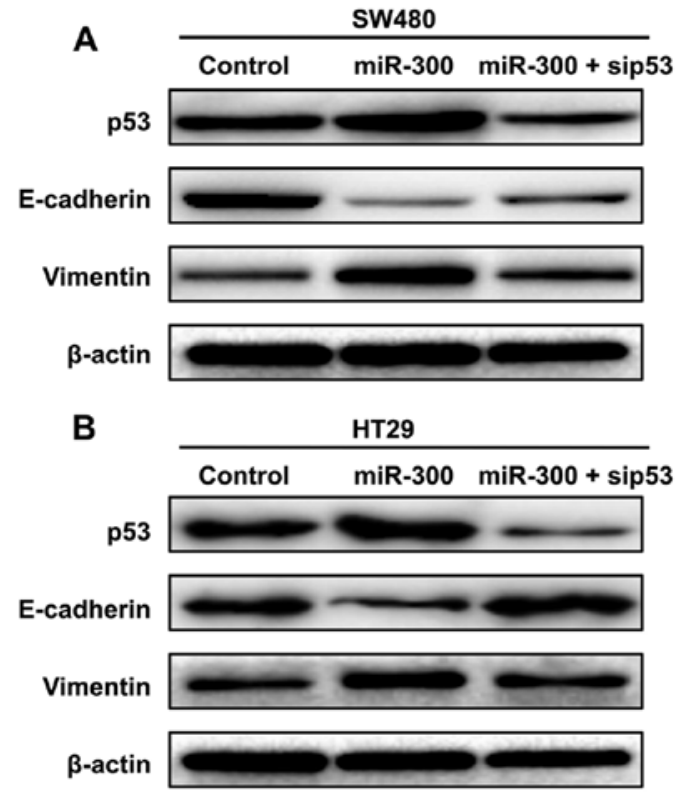

C

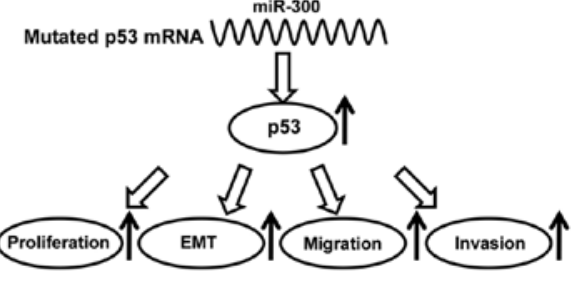

Figure 4. The expression level of p53 influences colorectal cancer (CRC) cell epithelial-mesenchymal transition (EMT) and a schematic model of miR-300 functions. (A) Western blot analysis was used to detect the level of p53, E-cadherin, and vimentin in SW480 cells transfected with miR-300 and further transfected with siRNAs to silence the expression of p53. $\beta$-actin was used as a protein-loading control. (B) Western blot analysis was used to detect the level of p53, E-cadherin, and vimentin in HT29 cells transfected with miR-300 and further transfected with siRNAs to silence the expression of p53. $\beta$-actin was used as a protein-loading control. (C) A schematic model of miR-300 functions in mutant p53-expressing human CRC. miR-300 increased the expression level of 53 by targeting the 53 promoter and promoted EMT, migration and invasion of CRC. 
A schematic model of miR-300 functions in mutant p53-expressing human CRC. As shown in Fig. 4C, miR-300 increased the expression level of p53 by targeting the p53 promoter and promoted EMT, migration and invasion of CRC. Specifically, miR-300 was found to be a direct positive regulator of p53 through binding to the binding site in 3'UTR of the p53 gene in human CRC cells. miR-300 promoted CRC cell proliferation, EMT, migration and invasion by targeting mutational p53 in vitro.

\section{Discussion}

miRNAs are small non-coding RNAs that silence mRNAs. In recent years, numerous studies have shown the effects of miRNAs on cancer-related proliferation, apoptosis, inflammation, migration, invasion and angiogenesis through regulation of the expression of target mRNAs (15-19). More than $60 \%$ of human protein-coding genes include conserved miRNAbinding sites and most protein-coding genes are regulated by miRNAs (20). Therefore, we hypothesized that there may be specific miRNAs which may directly regulate p53 expression. In the present study, we found that both miR-300 and p53 were significantly increased in CRC tissues relative to levels noted in the adjacent colorectal mucosal tissues. Both miR-300 and p53 were significantly correlated with lymphatic metastasis and TNM stage by clinicopathological analysis. Furthermore, we identified miR-300 as a direct positive regulator of p53 by binding to the 3'UTR of the human p53 gene.

It has been reported that the steps of metastasis involve the EMT process $(21,22)$. EMT is a process in which cells are transformed from an epithelial non-motile morphology to displaying mesenchymal characteristics, such a fibroblast shape and the enhanced ability of cell migration and invasion $(21,23,24)$. E-cadherin is related to tumor invasiveness, cancer metastasis, and poor prognosis $(25,26)$. The impact of E-cadherin on metastasis has been studied in various models (27-29). The interaction of E-cadherin molecules generates the core of the epithelial adherens junction (30). E-cadherin has been considered an epithelial marker. Vimentin regulates cell migration. Vimentin results in migration through recycling of endocytosed cell adhesion receptors (31). Vimentin regulates EMT induction and promotes migration in breast cancer (32). Vimentin has been considered a mesenchymal marker. In the present study, we found that the level of E-cadherin was decreased and the levels of the p53 and vimentin were increased after overexpression of miR-300. The level of E-cadherin was increased and the levels of the p53 and vimentin were decreased after silencing of p53 by siRNAs. Therefore, miR-300 and p53 induced CRC cell EMT. Furthermore, we aslso indicated that both miR-300 and p53 promoted CRC cell proliferation, migration and invasion in vitro.

In conclusion, we demonstrated that miR-300 is a direct positive regulator of $\mathrm{p} 53$ through binding to the binding site in the 3'UTR of the p53 gene in human CRC cells. Both miR-300 and p53 were significantly increased in CRC tissues. miR-300 was significantly correlated with lymphatic metastasis and TNM stage. Both miR-300 and p53 promoted $\mathrm{CRC}$ cell proliferation, migration and invasion in vitro. Taken together, we propose that miR-300 promotes proliferation and
EMT-mediated CRC migration and invasion by targeting p53. These findings lay the foundation for exploring the mechanism of CRC metastasis and seeking effective therapeutic targets.

\section{References}

1. Ferlay J, Soerjomataram I, Dikshit R, Eser S, Mathers C, Rebelo M, Parkin DM, Forman D and Bray F: Cancer incidence and mortality worldwide: sources, methods and major patterns in GLOBOCAN 2012. Int J Cancer 136: E359-E386, 2015.

2. Boyle P and Langman JS: ABC of colorectal cancer: Epidemiology. BMJ 321: 805-808, 2000.

3. Glade MJ: Food, nutrition, and the prevention of cancer: a global perspective. American Institute for Cancer Research/World Cancer Research Fund, American Institute for Cancer Research, 1997. Nutrition 15: 523-526, 1999.

4. Siegel R, Desantis C and Jemal A: Colorectal cancer statistics, 2014. CA Cancer J Clin 64: 104-117, 2014.

5. Muller PA and Vousden KH: Mutant p53 in cancer: New functions and therapeutic opportunities. Cancer Cell 25: 304-317, 2014.

6. Wang Y, Suh Y-A, Fuller MY, Jackson JG, Xiong S, Terzian T, Quintás-Cardama A, Bankson JA, El-Naggar AK and Lozano G: Restoring expression of wild-type p53 suppresses tumor growth but does not cause tumor regression in mice with a p53 missense mutation. J Clin Invest 121: 893-904, 2011.

7. Hollstein M, Sidransky D, Vogelstein B and Harris CC: p53 mutations in human cancers. Science 253: 49-53, 1991.

8. Creighton CJ, Gibbons DL and Kurie JM: The role of epithelialmesenchymal transition programming in invasion and metastasis: A clinical perspective. Cancer Manag Res 5: 187-195, 2013.

9. Lamouille S, Xu J and Derynck R: Molecular mechanisms of epithelial-mesenchymal transition. Nat Rev Mol Cell Biol 15: 178-196, 2014.

10. Sheppard D: Epithelial-mesenchymal interactions in fibrosis and repair. Transforming growth factor- $\beta$ activation by epithelial cells and fibroblasts. Ann Am Thorac Soc 12 (Suppl 1): S21-S23, 2015.

11. Kovacic JC, Mercader N, Torres M, Boehm M and Fuster V: Epithelial-to-mesenchymal and endothelial-to-mesenchymal transition: From cardiovascular development to disease. Circulation 125: 1795-1808, 2012.

12. Fraga CH, True LD and Kirk D: Enhanced expression of the mesenchymal marker, vimentin, in hyperplastic versus normal human prostatic epithelium. J Urol 159: 270-274, 1998.

13. Jiang L, Lai YK, Zhang J, Wang H, Lin MC, He ML and Kung HF: Targeting S100P inhibits colon cancer growth and metastasis by lentivirus-mediated RNA interference and proteomic analysis. Mol Med 17: 709-716, 2011.

14. Jiang L, Chen Y, Chan CY, Wang X, Lin L, He ML, Lin MC, Yew DT, Sung JJ, Li JC, et al: Down-regulation of stathmin is required for TGF-beta inducible early gene 1 induced growth inhibition of pancreatic cancer cells. Cancer Lett 274: 101-108, 2009.

15. Peacock O, Lee AC, Cameron F, Tarbox R, Vafadar-Isfahani N, Tufarelli $\mathrm{C}$ and Lund JN: Inflammation and MiR-21 pathways functionally interact to downregulate PDCD4 in colorectal cancer. PLoS One 9: e110267, 2014.

16. Zhang J, Fei B, Wang Q, Song M, Yin Y, Zhang B, Ni S, Guo W, Bian Z, Quan C, et al: MicroRNA-638 inhibits cell proliferation, invasion and regulates cell cycle by targeting tetraspanin 1 in human colorectal carcinoma. Oncotarget 5: 12083-12096, 2014.

17. Wang J, Paris PL, Chen J, Ngo V, Yao H, Frazier ML, Killary AM, Liu CG, Liang H, Mathy $\mathrm{C}$, et al: Next generation sequencing of pancreatic cyst fluid microRNAs from low grade-benign and high grade-invasive lesions. Cancer Lett 356: 404-409, 2015.

18. Kasinski AL and Slack FJ: Epigenetics and genetics. MicroRNAs en route to the clinic: Progress in validating and targeting microRNAs for cancer therapy. Nat Rev Cancer 11: 849-864, 2011.

19. Stahlhut C and Slack FJ: MicroRNAs and the cancer phenotype: Profiling, signatures and clinical implications. Genome Med 5: $111,2013$.

20. Friedman RC, Farh KK-H, Burge CB and Bartel DP: Most mammalian mRNAs are conserved targets of microRNAs. Genome Res 19: 92-105, 2009.

21. Christofori G: New signals from the invasive front. Nature 441: 444-450, 2006. 
22. Berx G, Raspé E, Christofori G, Thiery JP and Sleeman JP Pre-EMTing metastasis? Recapitulation of morphogenetic processes in cancer. Clin Exp Metastasis 24: 587-597, 2007.

23. Huber MA, Kraut $\mathrm{N}$ and Beug H: Molecular requirements for epithelial-mesenchymal transition during tumor progression. Curr Opin Cell Biol 17: 548-558, 2005.

24. Lee JM, Dedhar S, Kalluri R and Thompson EW: The epithelial-mesenchymal transition: New insights in signaling, development, and disease. J Cell Biol 172: 973-981, 2006.

25. Schipper JH, Frixen UH, Behrens J, Unger A, Jahnke K and Birchmeier W: E-cadherin expression in squamous cell carcinomas of head and neck: Inverse correlation with tumor dedifferentiation and lymph node metastasis. Cancer Res 51: 6328-6337, 1991.

26. Oka H, Shiozaki H, Kobayashi K, Inoue M, Tahara H, Kobayashi T, Takatsuka Y, Matsuyoshi N, Hirano S, Takeichi M, et al: Expression of E-cadherin cell adhesion molecules in human breast cancer tissues and its relationship to metastasis. Cancer Res 53: 1696-1701, 1993.

27. Perl A-K, Wilgenbus P, Dahl U, Semb H and Christofori G: A causal role for E-cadherin in the transition from adenoma to carcinoma. Nature 392: 190-193, 1998.
28. Vleminckx K, Vakaet L Jr, Mareel M, Fiers W and van Roy F: Genetic manipulation of E-cadherin expression by epithelial tumor cells reveals an invasion suppressor role. Cell 66: 107-119, 1991.

29. Frixen UH, Behrens J, Sachs M, Eberle G, Voss B, Warda A Löchner D and Birchmeier W: E-cadherin-mediated cell-cell adhesion prevents invasiveness of human carcinoma cells. J Cell Biol 113: 173-185, 1991.

30. Gumbiner BM: Regulation of cadherin-mediated adhesion in morphogenesis. Nat Rev Mol Cell Biol 6: 622-634, 2005.

31. Ivaska J, Vuoriluoto K, Huovinen T, Izawa I, Inagaki M and Parker PJ: PKCepsilon-mediated phosphorylation of vimentin controls integrin recycling and motility. EMBO J 24: 3834-3845, 2005.

32. Vuoriluoto K, Haugen H, Kiviluoto S, Mpindi JP, Nevo J, Gjerdrum C, Tiron C, Lorens JB and Ivaska J: Vimentin regulates EMT induction by Slug and oncogenic H-Ras and migration by governing Axl expression in breast cancer. Oncogene 30: 1436-1448, 2011. 